Ann. Biol. anim. Bioch. Biophys., I974, 14 (2), 239-250.

\title{
INFLUENCE D'UNE SUPPLÉMENTATION DE LA RATION EN SELS BILIAIRES CONJUGUÉS SUR L'ÉQUIPEMENT EN ACIDES BILIAIRES DE L'INTESTIN GRELLE DU RAT. MODIFICATIONS DE L'ABSORPTION APPARENTE DES ACIDES GRAS (1)
}

Y. DEMARNE*, E. SACQUET, Yveline VAN HEIJENOORT et Catherine MATHIS avec la collaboration technique de Marie-José Lecourtier

* Station de Recherches de Nutrition, Centre national de Recherches zootechniques, I. N. R. A., 78350 Jouy en Josas

Service des Animaux sans germes, Centre national de la Recherche scientifique, 91190 Gif sur Yvette

\section{RÉSUMÉ}

Une supplémentation de la ration, au taux de 5 p. I ooo, par une préparation de sels biliaires contenant principalement les sels de sodium des acides taurocholique $(78, \mathbf{1}$ p. Ioo) et taurodésoxycholique ( 7,5 p. IOo), pendant I 4 jours, permet de doubler la quantité de sels biliaires présente dans le tube digestif du Rat holoxénique en état de non réplétion. Cette augmentation (4o micromoles par intestin grêle) demeure cependant modérée en comparaison de l'importance du flux alimentaire de sels biliaires (I 25 micromoles par jour). Cela attire l'attention sur le rôle que joue l'absorption intestinale dans la régulation du pool des acides biliaires. Ce sont naturellement les composants qui sont présents dans l'aliment qui augmentent le plus au niveau intestinal (acides cholique et désoxycholique). Les quantités des autres acides biliaires sont diversement modifiées ; ainsi, celles de l'acide $\beta$-muricholique diminue peu, alors que celles de l'acide hyodésoxycholique diminue des $2 / 3$. Ces différentes observations sont discutées.

Cette supplémentation permet d'augmenter sensiblement l'utilisation digestive d'une matière grasse très riche en acides gras saturés (environ $43 \mathrm{p}$. Ioo d'acides palmitique et stéarique). L'absorption de ces acides gras est très fortement améliorée par rapport à ce qui est observé chez le rat non supplémenté. De plus, l'excrétion fécale de savons insolubles est très réduite.

Cette expérimentation permet donc de recréer artificiellement, et en présence d'une flore gastro-intestinale, les conditions remarquables d'absorption déjà étudiées en absence de flore.

(1) Ce travail a bénéficié d'une aide financière dans le cadre d'un contrat d'action thématique programmée du Centre national de la Recherche scientifique (thème : Écophysiologie des régulations). 


\section{INTRODUCTION}

L'utilisation digestive apparente des acides gras et principalement celle des acides gras à chaîne longue et saturée est influencée par la flore du tractus digestif. Le Rat axénique absorbe mieux les acides palmitique et stéarique que le Rat holoxénique. Parallèlement à cette augmentation de l'utilisation digestive apparente de ces acides gras, on observe une diminution de l'excrétion fécale de savons de calcium (DEmarnE: et al., 1970).

De nombreuses différences morphologiques et physiologiques existent entre Rats axéniques et holoxéniques et peuvent influer sur l'absorption intestinale des produits de l'hydrolyse des triglycérides alimentaires.

C'est ainsi que nous avons été amenés à étudier le rôle que pouvait jouer, dans ce phénomène, $1^{\prime}$ hypertrophie cæcale du Rat axénique. Nous avons pu montrer que cette importante modification morphologique ne pouvait pas expliquer, à elle seule, les différences existant au niveau de l'absorption des acides gras en présence et en absence de flore intestinale (DEMARne et al., I972).

De plus, le tube digestif du Rat axénique contient 2 à 3 fois plus de sels biliaires que celui du Rat holoxénique, et la composition en acides biliaires n'est pas la même dans les deux types d'animaux. En l'absence de flore, les métabolites bactériens sont absents et les proportions d'acides biliaires primaires ne sont pas les mêmes (SACQUET, I97I ; SacqueT, Garnier et IONESCo, I970; Van HeIJENoOrT et al., I972).

Compte tenu du rôle que jouent les sels biliaires conjugués dans les phénomènes de solubilisation micellaire des acides gras au niveau intestinal, nous nous sommes proposés de rechercher si les modifications rapportées pour l'absorption n'étaient pas liées aux différences quantitative et qualitative de l'équipement en sels biliaires des deux types d'animaux.

Dans cette étude notre but a été double :

- augmenter artificiellement la teneur en sels biliaires dans le tractus digestif du Rat holoxénique par supplémentation alimentaire, et étudier les modifications de la teneur et de la composition en sels biliaires au niveau de l'intestin grêle ;

- mesurer l'influence de cette supplémentation sur l'utilisation digestive apparente des acides gras.

Cette étude est effectuée au moyen de deux expériences, les exigences de l'une n'étant pas compatibles avec celles de l'autre.

\section{I. - MATÉRIEL ET MÉTHODES}

\section{Protocole expérimental}

Les expériences sont réalisées avec des rats holoxéniques adultes de souche Fischer. Les animaux reçoivent pendant quatorze jours un régime dont la composition est rapportée dans le tableau I. La matière grasse utilisée dans la fabrication du régime est un mélange de beurre de cacao, huile de coton et huile de coprah (50/25/25). La composition en acides gras de ce mélange est rapportée dans le tableau 2. 
Pour les animaux soumis à une supplémentation en sels biliaires, on introduit dans le régime une préparation commerciale de taurocholate de sodium (1) au taux de 5/I ooo par rapport à la matière sèche. L'analyse de cette préparation (voir techniques d'analyse) fait apparaître qu'outre le taurocholate elle contient d'autres sels biliaires. La composition molaire de ce mélange est rapportée dans le tableau 3 .

TABLEAU I

Composition du régime (p. Ioo en poids)

\begin{tabular}{|c|c|}
\hline Caséine $\ldots \ldots \ldots \ldots \ldots \ldots \ldots \ldots \ldots \ldots \ldots$ & 17,7 \\
\hline 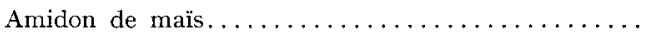 & 56,7 \\
\hline Cellulose $\ldots \ldots \ldots \ldots \ldots \ldots \ldots \ldots \ldots \ldots$ & 5,4 \\
\hline Sels $\min e ́ r a u x$ & 4,8 \\
\hline Vitamines et acides aminés complémentaires ...... & 2,1 \\
\hline 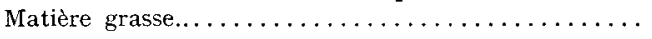 & 13,3 \\
\hline
\end{tabular}

TABLEAU 2

Composition en acides gras de la matière grasse ingérée

Acides gras (p. 100 des esters méthyliques dosés)

\begin{tabular}{c|c|c|c|c|c}
$\mathrm{C}_{12}$ & $\mathrm{C}_{14}$ & $\mathrm{C}_{16}$ & $\mathrm{C}_{18}$ & $\mathrm{C}_{18: 1}$ & $\mathrm{C}_{18: 2}$ \\
\hline 15,5 & 6,2 & 23,6 & 19,8 & 29,3 & 5,7 \\
\hline
\end{tabular}

\section{TABLEAU 3}

Composition molaive du mélange de sels biliaires

\begin{tabular}{|c|c|}
\hline Sels biliaires & $\begin{array}{l}\text { Concentration } \\
(\text { p. 100) }\end{array}$ \\
\hline Cholate $(3 \alpha, 7 \alpha, 12$ trihydroxy $5 \beta$ cholanate). & 78,1 \\
\hline 7 céto- $3 \alpha, 12 \alpha$, dihydroxy $5 \beta$ cholanate $\ldots$ & 1,1 \\
\hline Désoxycholate $(3 \alpha, 12 \alpha$, dihydroxy $5 \beta$ cholanate) & 17,5 \\
\hline Chénodésoxycholate ( $3 \alpha, 7 \alpha$, dihydroxy $5 \beta$ cholanate) . & 1,8 \\
\hline Lithocholate ( $3 \alpha$, hydroxy $5 \beta$ cholanate). & 1,4 \\
\hline
\end{tabular}

- Dans l'expérience $\mathbf{x}$, on se propose de déterminer dans quelle mesure la supplémentation en sels biliaires de la ration modifie l'équipement en sels biliaires au niveau de l'intestin grêle.

Trois rats reçoivent, pendant quatorze jours, le régime supplémenté en sels biliaires. Au matin du quinzième jour, ces animaux sont sacrifiés en état de non réplétion et les intestins grêles, vides de toute trace d'aliment, sont prélevés. De la même façon, on procède au prélèvement des intes-

(1) Taurocholate de sodium Stéraloïds Inc. Pawling, N. Y., Batch 7076. 
tins grêles de six autres rats ayant reçu, pendant la même période que précédemment, le même régime mais non supplémenté. Au moment du sacrifice, les rats ayant été supplémentés en sels biliaires pèsent en moyenne $280 \mathrm{~g}$ et sont âgés d'environ 95 jours. Les autres rats pèsent en moyenne $266 \mathrm{~g}$ et sont âgés de 90 jours.

- Dans l'expérience 2, on se propose de déterminer quelles modifications de l'utilisation digestive des matières grasses entraîne la supplémentation en sels biliaires de la ration.

Six rats âgés de 58 jours et pesant en moyenne $255 \mathrm{~g}$ en début d'expérience sont répartis en deux lots homogènes $\mathrm{A}$ et $\mathrm{B}$ et sont disposés en cages individuelles. Tous ces animaux reçoivent pendant $\mathrm{I}_{4}$ jours le même régime de base, mais pour les rats du lot $\mathrm{A}$, le régime est supplémenté en sels biliaires au taux de $5 \mathrm{p}$. I ooo.

Cette expérience 2 se déroule dans des conditions semblables à celles de l'expérience I. Les rats sont issus, dans les deux cas, de la même salle d'élevage et toutes les expériences se déroulent dans cette salle.

\section{Méthodes d'analyse}

\section{Dosage des sels biliaires au niveau de l'intestin grêle.}

Pour éviter les erreurs provenant de ce qu'il est difficile de prélever correctement le contenu de l'intestin grêle du rat, la totalité de cet organe, contenu et contenant, est prélevée, broyée et soumise à l'analyse.

Comme des essais préliminaires ont montré que l'état de réplétion au cours de la digestion était très irrégulier, les rats sont sacrifiés en dehors des périodes de digestion, le matin à ro heures.

La méthode d'analyse et de dosage tient compte de ce que la séparation et le dosage des diverses espèces chimiques de sels biliaires s'accompagnent de pertes qu'il est nécessaire de pouvoir apprécier non seulement pour l'ensemble des sels biliaires mais aussi pour chaque espèce chimique. Elle consiste à introduire dans la préparation soumise à l'analyse une quantité connue de sels biliaires $4^{-14} \mathrm{C}$; et après séparation, à doser les sels biliaires en considérant que les acides marqués au carbone $\mathrm{I}_{4}$ subissent au cours de l'analyse les mêmes pertes que leurs homologues non marqués.

La préparation de sels biliaires $4^{-14} \mathrm{C}$ est constituée par un extrait éthanolique de bile de Rat holoxénique ayant reçu du cholestérol $4^{-14} \mathrm{C}$ dont la composition a fait l'objet de déterminations précises et renouvelées.

Les rats sont anesthésiés à l'éther, les intestins sont prélevés, rassemblés, broyés dans l'éthanol et soumis à trois extractions éthanoliques à reflux pendant 48 heures. Ces extraits sont traités par la technique de Grundy et al. (1965) et les esters méthyliques sont soumis à deux types de chromatographie sur gel de silice $G$, en couches minces :

a) une fraction est soumise à une chromatographie ascendante sur plaque $20 \times 20 \times 0,025$ $\mathrm{cm}$, d'abord par le benzène, puis par le mélange chloroforme/méthanol/ammoniaque I $2 \mathrm{~N}: 90 / 45 / \mathbf{I o}$ selon Subbiah et Kuksis (1968) ;

b) une fraction est soumise à une chromatographie descendante sur plaque $40 \times 20 \times 0,05 \mathrm{~cm}$, d'abord par le benzène, puis par le mélange chloroforme/acétone/méthanol : 70/25/5 (Hoffmann, rg64).

La technique A rassemble les esters méthyliques sur une étroite bande de gel : elle permet de vérifier que la méthylation est totale et d'effectuer un dosage de la quantité totale des sels biliaires.

La technique $B$ permet de séparer les esters méthyliques des acides cholique, $\beta$-muricholique, hyodésoxycholique, 7 -céto-3,12-dihydroxy-5-cholanique, chénodésoxycholique, désoxycholique, ursodésoxycholique, d'autres acides dihydroxylés indéterminés, de l'acide lithocholique et de del1x composés indéterminés voisins de celui-ci. La fraction qui contient l'adide cholique contient aussi l'acide $\alpha$-muricholique et un autre acide trihydroxylé inconnu, qui ont toujours été trouvés en faible quantité (de l'ordre de 2 p. Ioo de l'ensemble des acides biliaires) quand ils ont été dosés.

Après élution par le méthanol, les différentes fractions font l'objet d'une part, d'une mesure de la radioactivité par mesure en scintillation liquide dans le mélange diméthyl-POPOP : O, $\mathbf{~ g}$, $\mathrm{PPO}: 0,04 \mathrm{~g}$, toluène : I litre, d'autre part, d'une mesure de la masse des sels biliaires par dosage enzymatique par la 3-hydroxy-stéroïde-déhydrogénase selon Sidbury (1964).

Cette méthode permet de doser les sels biliaires avec une précision qui est satisfaisante : l'écart entre les valeurs extrêmes dans une série de dosages d'un même échantillon est de io p. Ioo. La radioactivité retrouvée en fin d'expérience est supérieure à 90 p. Ioo de la radioactivité introduite. L'inconvénient de cette méthode est qu'elle est très longue et qu'en conséquence les dosages ne peuvent être faits que sur le pool des extraits éthanoliques et non pas sur les extraits obtenus à partir de chaque prélèvement. 
Analyse des aliments et des fèces.

Les techniques d'extraction et d'analyse des acides gras des fèces et des régimes ainsi que la méthodologie de la détermination des coefficients d'utilisation digestive ont été décrites précédemment (Demarne et al., 1970).

Les teneurs en calcium des matières fécales ont été obtenues par spectrophotométrie de fiamme après minéralisation des échantillons selon la technique de GUEGUEN et Rombauts (196r).

\section{II. - RÉSULTATS}

\section{I. - Variations de l'équipement en sels biliaires (expérience I)}

La consommation d'aliment est semblable chez les rats qui reçoivent des sels biliaires et chez ceux qui n'en reçoivent pas ( $185 \mathrm{~g}$ et $\mathrm{I} 85,3 \mathrm{~g}$ ). Les animaux supplémentés ingèrent en moyenne $\mathbf{2} 33$ micromoles de sels biliaires par jour et par rat, soit I 720 micromoles pendant les I4 jours d'expérience.

Sous 1'influence de cet apport, la quantité totale de sels biliaires par intestin grêle de rat, qui est de 48 micromoles chez les rats holoxéniques recevant un régime normal, s'élève à 88 micromoles en fin d'expérience.

La figure ci-contre représente les quantités des différents sels biliaires exprimées en micromoles par intestin grêle de rat. L'acide cholique et 1'acide désoxycholique, qui représentent respectivement 78 p. Ioo et I 8 p. Ioo environ de l'apport alimentaire

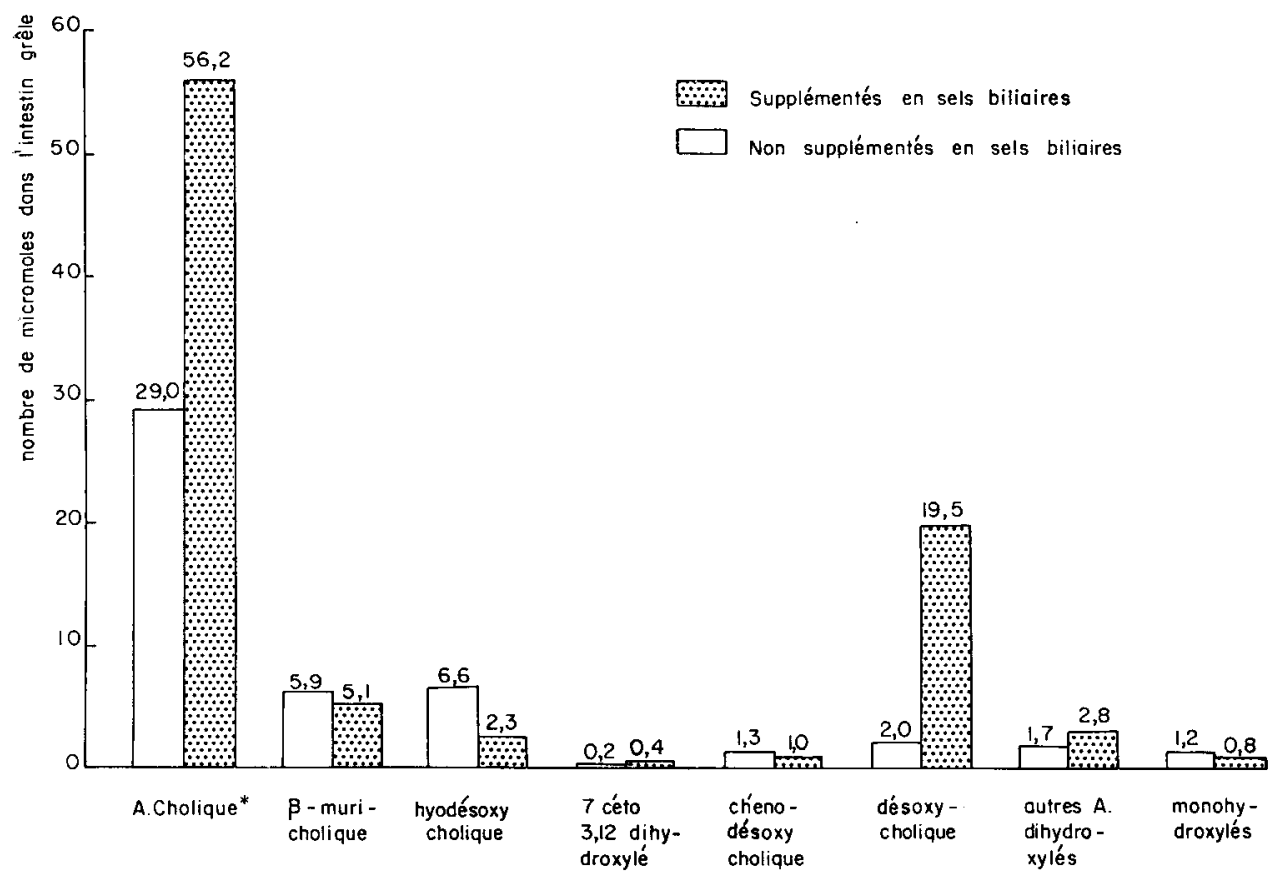

* Cette fraction contient des traces d'acide a-muricholique et $d$ 'un acide trihydroxylé inconnu.

FIG. I. - Quantités des différents acides biliaires obtenus dans chaque lot (valeurs exprimées en micromoles/intestin grêle). 
des acides biliaires, augmentent d'une manière très importante et constituent respectivement $64 \mathrm{p}$. Ioo et $22 \mathrm{p}$. Ioo de la quantité totale des acides biliaires mesurée au niveau de 1'intestin grêle. Parmi les autres espèces chimiques d'acides biliaires, il convient de remarquer que l'acide $\beta$-muricholique diminue peu (témoin 5,9 micromoles, supplémentés : 5 , I micromoles, soit une diminution de $I / 7^{\text {e }}$ de la valeur $\mathrm{du}$ témoin), alors qu'au contraire l'acide hyodésoxycholique diminue d'une manière très considérable (témoins : 6,6 micromoles, supplémentés : 2,3 micromoles), soit une diminution des $2 / 3$ de la valeur des témoins).

\section{2. - Bilans digestifs (expérience 2)}

La consommation d'aliment n'est pas sensiblement influencée par la supplémentation en sels biliaires. Le tableau 4 montre que l'utilisation digestive apparente de la matière grasse est meilleure dans le lot $\mathrm{A}$ (supplémenté) que dans le lot $\mathrm{B}$. Cette amélioration de la digestibilité de la matière grasse suffit à faire varier légèrement celle de l'ensemble de la ration. L'utilisation digestive de la fraction non lipidique du régime, obtenue par le calcul, n'est pas affectée par la supplémentation en sels biliaires.

\section{TABLEAU 4}

Bilans digestifs globaux (valeurs moyennes et extrêmes)

\begin{tabular}{c|c|c|c|c}
\hline Lots & MSI (g) (1) & $\begin{array}{c}\text { CUDa MS } \\
(\%)\left({ }^{2}\right)\end{array}$ & $\begin{array}{c}\text { CUDa MG } \\
(\%)\left({ }^{3}\right)\end{array}$ & $\begin{array}{c}\text { CUDa MSNL } \\
(\%)\left({ }^{4}\right)\end{array}$ \\
\hline A (+) & $\begin{array}{c}196,0 \\
(191,4-199,5)\end{array}$ & $\begin{array}{c}87,5 \\
(86,7-88,0)\end{array}$ & $\begin{array}{c}92,7 \\
(91,4-93,9)\end{array}$ & $\begin{array}{c}86,7 \\
(85,9-87,3)\end{array}$ \\
\hline B & $\begin{array}{c}191,0 \\
(186,7-194,8)\end{array}$ & $\begin{array}{c}85,9 \\
(85,2-8(6,7)\end{array}$ & $\begin{array}{c}83,0 \\
(80,7-84,7)\end{array}$ & $\begin{array}{c}86,3 \\
(85,9-87,9)\end{array}$ \\
\hline
\end{tabular}

(1) MSI : matière sèche ingérée.

(2) CUDa MS : digestibilité apparente de la ration.

( $\left.{ }^{3}\right)$ CUDa MG : digestibilité apparente de la matière grasse.

(4) CUDa MSNL : digestibilité apparente de la fraction non lipidique de la ration.

Si les matières sèches fécales contiennent 7,5 p. Ioo de lipides dans le lot $\mathrm{A}$, elles en contiennent I5 p. roo dans le lot B. De plus, les lipides fécaux se répartissent différemment entre les fractions solubles (S) et insolubles (SI), selon que la ration a été ou non supplémentée en sels biliaires. Dans le lot $A$, environ 60 p. Ioo des matières grasses excrétées se trouvent dans la fraction soluble, alors que dans le lot B, les proportions sont inversées (tab1. 5).

L'augmentation de l'excrétion fécale de lipides sous forme insoluble ne peut être associée à une diminution de l'utilisation digestive du calcium ou à un accroissement de son excrétion endogène. Fin effet, les quantités de calcium excrétées, rappor- 
tées par gramme d'aliment ingéré, sont identiques dans les deux lots. La synthèse de savons de calcium, constituants essentiels des fractions insolubles des lipides fécaux, n'a donc pu, en aucun cas, être limitée par la disponibilité en ions $\mathrm{Ca}^{++}$dans le milieu.

\section{TABLEAU 5}

Excrétion fécale de lipides et de calcium (valeurs moyennes et extrêmes)

\begin{tabular}{|c|c|c|c|c|c|c|}
\hline \multirow{2}{*}{ Lots } & \multirow{2}{*}{$\operatorname{MSF}^{*}(g)\left({ }^{1}\right)$} & \multicolumn{2}{|c|}{ FS $\left({ }^{2}\right)$} & \multicolumn{2}{|c|}{$\mathrm{FI}\left({ }^{3}\right)$} & \multirow{2}{*}{$\mathrm{Ca}^{++} \mathrm{E}(4)$} \\
\hline & & $(\%)$ & (g) & $(\%)$ & (g) & \\
\hline$A(+)$ & $\begin{array}{c}24,6 \\
(23,6-25,5)\end{array}$ & $\begin{array}{c}4,6 \\
(3,9-5,1)\end{array}$ & $\begin{array}{c}1,1 \\
(0,9-1,3)\end{array}$ & $\begin{array}{c}2,9 \\
(2,5-3,3)\end{array}$ & $\begin{array}{c}0,7 \\
(0,6-0,8)\end{array}$ & $\begin{array}{c}11,41 \\
(11,10-11,57)\end{array}$ \\
\hline B & $\begin{array}{c}26,9 \\
(25,9-28,3)\end{array}$ & $\begin{array}{c}6,1 \\
(5,6-6,5)\end{array}$ & $\begin{array}{c}1,6 \\
(1,5-1,8)\end{array}$ & $\begin{array}{c}9,6 \\
(8,4-10,8)\end{array}$ & $\begin{array}{c}2,6 \\
(2,2-3,1)\end{array}$ & $\begin{array}{c}11,43 \\
(10,95-11,90)\end{array}$ \\
\hline
\end{tabular}

(1) MSF : matière sèche fécale.

(2) FS : fraction soluble des lipides fécaux.

( $\left.{ }^{3}\right) \mathrm{FI}$ : fraction insoluble des lipides fécaux.

(4) $\mathrm{Ca}^{++} \mathrm{E}:$ excrétion de calcium (mg/g d'aliment ingéré).

Sur le tableau 6 , on notera que les compositions moyennes en acides gras des fractions solubles et insolubles des matières grasses fécales sont très comparables dans le lot ayant reçu une supplémentation en sels biliaires (lot A). Il n'en est pas de même dans le lot $B$. Dans ce cas, la teneur relative en acides gras saturés et longs $\left(\mathrm{C}_{16}\right.$ et $\left.{ }_{18}\right)$ est plus importante dans la fraction insoluble que dans la fraction soluble. Cette observation va de pair avec une plus faible concentration en acide oléique dans la fraction insoluble.

\section{TABLEAU 6}

Composition en acides gras des fractions solubles (FS) et insolubles (FI)

(p roo des esters méthyliques dosés)

\begin{tabular}{|c|c|c|c|c|c|c|c|c|}
\hline Lots & Fractions & $c_{12}$ & $\mathrm{C}_{14}$ & $\mathrm{C}_{16}$ & $\mathrm{C}_{18}$ & $C_{18: 1}$ & $C_{18: 2}$ & $?$ \\
\hline \multirow{2}{*}{$A(+)$} & FS & 1,8 & 4,1 & 40,2 & 39,3 & 11,4 & 3,2 & - \\
\hline & FI & 2,5 & 4,3 & 39,9 & 43,3 & 8,3 & 1,3 & - \\
\hline \multirow{2}{*}{ B } & $\mathrm{FS}$ & 5,0 & 6,3 & 37,9 & 32,3 & 15,5 & 3,0 & 1,3 \\
\hline & FI & 2,0 & 4,3 & 42,8 & 45,0 & 4,2 & 1,0 & 1,0 \\
\hline
\end{tabular}


L'utilisation digestive apparente des acides gras à chaîne moyenne $\left(C_{12}\right)$ ou à chaîne longue et insaturée $\left(\mathrm{C}_{18: 1}, \mathrm{C}_{18: 2}\right)$ ne semble pas être notablement influencée par la supplémentation en sels biliaires de la ration. En revanche, celle des acides gras à chaîne longue et saturée $\left(\mathrm{C}_{14}, \mathrm{C}_{16}\right.$ et $\left.\mathrm{C}_{18}\right)$ est sensiblement améliorée chez les animaux ayant subi cette supplémentation (tabl. 7).

TABLEAU 7

Utilisation digestive apparente des acides gras (p. 100)

\begin{tabular}{c|c|c|c|c|c|c}
\hline \hline Lots & $\mathrm{C}_{12}$ & $\mathrm{C}_{14}$ & $\mathrm{C}_{\mathbf{1 6}}$ & $\mathrm{C}_{\mathbf{1 8}}$ & $\mathrm{C}_{\mathbf{1 8}: 1}$ & $\mathrm{C}_{\mathbf{1 8}: \mathbf{2}}$ \\
\hline $\mathrm{A}(+)$ & 99,1 & 95,9 & 89,6 & 89,5 & 97,9 & 97,2 \\
\hline $\mathrm{B}$ & 96,1 & 86,3 & 70,3 & 64,9 & 95,5 & 95,2 \\
\hline \hline
\end{tabular}

\section{III. - DISCUSSION}

Dans nos expériences, le prélèvement des intestins grêles, pour doser les sels biliaires, est effectué en dehors des périodes de réplétion digestive. Les valeurs ainsi mesurées constituent des minima qui correspondent aux quantités maintenues par le cycle entérohépatique au moment du sacrifice de l'animal, auxquelles il convient d'ajouter, pendant les périodes de réplétion, les quantités correspondant à l'aliment en cours de digestion.

Sous l'influence de l'apport alimentaire la quantité totale de sels biliaires contenue dans l'intestin grêle de ces rats holoxéniques, s'élève et se rapproche de celle précédemment observée chez les rats axéniques : 88 micromoles chez ces rats holoxéniques, IOO à IIO micromoles chez les rats axéniques (VAN HEIJENOORT et al., I972).

Les quantités de sels biliaires excrétées au niveau des fèces n'ont pas été dosées. Cependant, chez les rats holoxéniques qui reçoivent des sels biliaires dans leur aliment, les quantités présentes au niveau de 1'intestin grêle ( 88 micromoles) demeurent très faibles en comparaison de la quantité totale de sels biliaires ingérée pendant la durée de l'expérience (I 720 micromoles/rat). L'excrétion totale de sels biliaires pendant cette période est donc voisine de l'ingestion. Cette excrétion est très supérieure à celle des rats qui ne reçoivent pas de sels biliaires dans l'aliment. Celle-ci varie selon les auteurs. D'après KELLOG (I97I), elle est de l'ordre de 2 micromoles chez les rats axéniques pour Ioo $\mathrm{g}$ de poids corporel et par jour, et de 4 micromoles chez les rats holoxéniques. En admettant que pendant la période d'expérience, l'excrétion soit uniforme, celle-ci serait, d'après le calcul, de 40 micromoles/j/Ioo g de poids vif, soit Io fois l'excrétion des sujets holoxéniques non supplémentés.

Ces valeurs, relativement basses du pool intestinal des sels biliaires par comparaison au flux d'origine alimentaire, attirent l'attention sur l'importance que jouent les mécanismes d'absorption et plus particulièrement sur le rôle de l'absorption passive, dans la limitation de ce pool. Celui-ci dépend de la quantité de sels biliaires qui 
est maintenue par le cycle entérohépatique et donc de l'absorption intestinale. Il est admis que l'absorption des sels biliaires se fait selon un mécanisme d'absorption active qui, en raison de la valeur très basse $\mathrm{du} \mathrm{Km}$ se fait toujours à la vitesse maximale, et deux mécanismes d'absorption passive dont l'activité est fonction de la concentration (Schiff, Smal, et Diftschy, I972). Il est donc vraisemblable que la quantité de sels biliaires présente au niveau de l'intestin grêle dépend essentiellement de l'absorption passive qui augmente quand la concentration des sels biliaires s'élève et joue ainsi un rôle régulateur du pool.

La composition des sels biliaires varie sous l'influence de l'apport alimentaire. En dehors de l'augmentation du cholate et du désoxycholate les variations des quantités des sels des acides $\beta$-muricholique et hyodésoxycholique méritent quelques commentaires : l'acide $\beta$-muricholique diminue très peu, alors que l'acide hyodésoxycholique diminue d'une manière très importante.

Les bactéries intestinales jouent un rôle dans la formation de l'acide hyodésoxycholique, car cet acide biliaire n'existe pas chez le Rat axénique (SACQUET, GARNIER, IonEsco, I97o). Chez celui-ci, il n'existe pas non plus d'acide hyocholique, ce qui exclut que la formation de l'acide hyodésoxycholique chez le Rat holoxénique se fasse par déshydroxylation en 7 de l'acide hyocholique, processus par lequel la flore bactérienne forme 1'acide désoxycholique à partir de l'acide cholique et 1'acide lithocholique à partir de l'acide chénodésoxycholique. Chez le Rat holoxénique, il est très probable que l'acide hyodésoxycholique se forme selon le schéma donné par EinaRsSON (I966) :

acide chénodésoxycholique $(3 \alpha, 7 \alpha$-dihydroxy-5 $\beta$-cholanique) $\stackrel{\text { bactéries }}{\longrightarrow}$ acide litho-

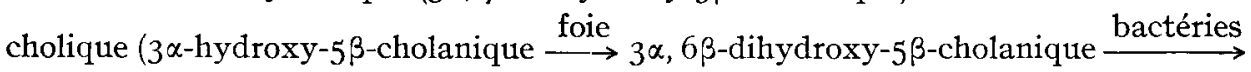
$3 \alpha$-hydroxy-6-céto- $5 \beta$-cholanique $\stackrel{\text { bactéries }}{\longrightarrow}$ hyodésoxycholique $(3 \alpha, 6 \alpha$-dihydroxy$5 \beta$-cholanique).

Dans la présente expérience, la diminution de l'acide hyodésoxycholique pourrait avoir pour origine une diminution du nombre ou de l'activité métabolique des bactéries qui participent à la formation de l'acide hyodésoxycholique. Il est possible que cette diminution soit causée par 1'acide désoxycholique qui augmente de 2 à I9,5 micromoles sous l'influence de l'apport alimentaire, et dont le pouvoir antibactérien est démontré in vitro mais reste à établir in vivo (ShImada, SUTTER, FineGold, I970).

Quant à l'absence de diminution importante de l'acide $\beta$-muricholique, elle peut s'expliquer de deux manières. On sait que cet acide biliaire est le produit terminal d'une chaîne métabolique à laquelle appartiennent l'acide chénodésoxycholique et l'acide $\alpha$-muricholique. La préparation commerciale des sels biliaires introduite dans le régime alimentaire ne contient aucun des éléments de cette chaîne métabolique, hormis une très faible quantité d'acide chénodésoxycholique et de son métabolite bactérien, l'acide lithocholique. La présence de ces composés dans l'aliment n'augmente d'ailleurs pas leur quantité au niveau de l'intestin grêle des rats supplémentés. Pour expliquer la faible diminution de l'acide $\beta$-muricholique, il faudrait admettre que la synthèse de cet acide biliaire n'est guère modifiée par l'apport alimentaire des sels biliaires. Cette hypothèse, si elle se révélait exacte, pourrait être étendue de l'acide $\beta$-muricholique à l'ensemble des acides biliaires. Il est admis, en effet, que la rétroinhibition de la synthèse des différents acides biliaires s'exerce à un stade initial de 
leur formation, avant qu'ils ne soient différenciés et, en conséquence, est égale pour chacun d'eux (SHEFER et al., I969). Mais il est aussi possible que la plus faible formation d'acide hyodésoxycholique chez les sujets de cette expérience laisse disponible davantage d'acide hydroxylé en $3 \alpha$ et $6 \beta$ pour la synthèse de l'acide $\beta$-muricholique.

La supplémentation en sels biliaires de la ration améliore l'utilisation digestive de la matière grasse ingérée. C'est au niveau des acides gras saturés (palmitique et stéarique, $\mathrm{C}_{16}$ et $\mathrm{C}_{18}$ ) que l'on observe les augmentations d'absorption les plus significatives. De plus, cette supplémentation provoque une diminution importante de l'excrétion de savons insolubles, alors que 1'excrétion de $\mathrm{Ca}^{++}$n'est pas modifiée.

Il semble donc que les différences rapportées précédemment entre rats axéniques et rats holoxéniques pour ce qui concerne l'absorption intestinale des lipides (DEMARNE et al., I970) peuvent être recréées en présence de flore gastro-intestinale, à partir du moment où il est possible d'augmenter artificiellement la teneur en sels biliaires au niveau de l'intestin grêle. On peut donc penser que l'augmentation de l'absorption des matières grasses enregistrée en absence de flore est en grande partie due au fait que, chez le Rat, l'état axénique multiplie par 2 ou 3 la teneur en sels biliaires dans l'intestin.

Dans tous les cas, l'amélioration de l'utilisation digestive des matières grasses est toujours liée à une augmentation de 1'absorption des acides gras saturés, et à une diminution de 1'excrétion de savons de calcium.

Comment une augmentation de la teneur en sels biliaires dans l'intestin grêle peut-elle influencer ces facteurs?

On sait qu'à même concentration en sels biliaires conjugués dans le milieu, la solubilisation micellaire des acides gras longs et saturés est beaucoup plus faible que celle des acides gras longs insaturés (Constantin et SAvary, I965 ; Savary et ConsTANTIN, I966). In vitro, quand on augmente la concentration en sels biliaires, on provoque une augmentation parallèle de la solubilisation micellaire de l'acide palmitique et de la $\beta$-monooléine. De plus, dans un système sels biliaires- $\beta$-monooléine-acide palmitique quand on augmente la concentration en $\beta$-monooléine, on augmente la quantité d'acide palmitique solubilisée (HoFmanN, Ig6I).

Dans toutes nos expériences (DEMARNE et al., r970, I972), nous avons employé des matières grasses très riches en acides gras saturés (environ $45 \mathrm{p}$. Ioo des acides gras dosés). On peut donc concevoir que la solubilisation micellaire des acides palmitique et stéarique a été meilleure chez les rats axéniques ou holoxéniques supplémentés en sels biliaires que chez les rats holoxéniques non supplémentés. De plus, quand le rapport acides gras longs saturés/acide oléique dans la ration augmente, les améliorations de l'absorption des acides gras saturés liées à l'état axénique sont plus faibles (DEMARne et al., I970, 1972). Ceci semblerait démontrer une influence concomitante de la teneur en sels biliaires et de la concentration en acide oléique dans la ration sur l'amélioration de 1'absorption des acides palmitique et stéarique. Il nous paraît donc difficile d'expliquer ce phénomène autrement que par une amélioration de la solubilisation micellaire des acides gras longs et saturés.

Quand on ajoute des ions $\mathrm{Ca}^{++}$à une solution micellaire d'acides gras longs saturés dans des sels biliaires conjugués, les acides gras quittent la phase micellaire pour précipiter sous forme de savons insolubles. Quand ces mêmes acides gras sont solubilisés dans des micelles mixtes contenant de la $\beta$-monooléine, le précipité ne se forme plus. Les micelles mixtes, qui sont normalement présents au niveau du jéjunum seraient 
une forme de protection efficace contre la précipitation des acides gras saturés par les ions $\mathrm{Ca}^{++}$du milieu (HoFmanN, I963). Une augmentation de la teneur en sels biliaires dans l'intestin grêle doit conduire à une augmentation du rapport

$$
\frac{\text { lipides sous forme micellaire }}{\text { lipides sous forme émulsionnée }} \text {. }
$$

Cet effet, d'après BorGs'rRöm ( I967), résulte d'une augmentation du volume micellaire et non d'une augmentation de la concentration en lipides dans la phase micellaire. Une plus grande capacité de solubilisation micellaire peut entraîner une augmentation de la vitesse de transfert des acides gras saturés de la phase "émulsion " où a lieu la lipolyse à la phase " micelle mixte " avec les sels biliaires conjugués et la $\beta$-monooléine. De ce fait, ces acides gras saturés seraient plus rapidement et plus efficacement protégés de la précipitation par les ions $\mathrm{Ca}^{++}$. Ceci expliquerait pourquoi le Rat axénique et le Rat holoxénique supplémentés en sels biliaires excrètent moins de savons de calcium que le Rat holoxénique. Ce serait donc l'ensemble de ces phénomènes qui contribuerait à différencier l'absorption des acides gras saturés chez le Rat axénique et le Rat holoxénique.

Reçu pour publication en août 1973.

\title{
REMERCIEMENTS
}

Nous exprimons nos remerciements à. M. le Professeur Chevalliter et à MM. Luton et Mathe grâce à la collaboration desquels le dosage de la radioactivité des sels biliaires a pu être effectué.

Nous tenons également à remercier Mme ChÉMEnt et M. le Professeur SaRLes qui ont bien voulu relire notre manuscrit et nous faire part de leurs suggestions.

\section{SUMMARY}

\author{
EFFECT OF CONJUGATED BILE SAIT SUPPLEMENTATION
}

OF THE RATION ON AMOUNT OF BILE ACID IN THE SMALI INTESTINE OF RAT. CHANGES IN APPARENT FATTY ACID ABSORPTION

When $5 \mathrm{p}$. Ioo of the ration is supplemented for $\mathrm{I}_{4}$ days with bile salts mostly containing sodium salts from taurocholic (78.I p. Ioo) and taurodeoxycholic (17.5 p. roo) acids, the amount of bile salts present is doubled in the digestive tract of the holoxenic rat in a state of non-repletion. This increase (40 micromoles per small intestine) remains moderate as compared to the amount of food flux bile salt ( 25 micromoles per day). This draws attention to the role played by intestinal absorption in regulating the bile acid pool. The components present in the food naturally increase most in the intestine (cholic and deoxycholic acids). Quantities of other bile acids are modified in various ways; thus, those of $\beta$-muricholic acid decrease little, while those of hyodeoxycholic acid decrease by $2 / 3$. These different observations are discussed.

Using this supplementation markedly increases digestive utilization of fatty matter very rich in saturated fatty acids (about $43 \mathrm{p}$. roo palmitic and stearic acids). Absorption of these fatty acids is greatly improved as related to that observed in the non-supplemented rat. Moreover, fecal excretion of insoluble soap is very reduced.

The remarkable absorption conditions already studied in absence of flora, can be artificially reproduced in presence of gastro-intestinal flora in this experiment. 


\section{RÉFÉRENCES BIBLIOGRAPHIQUES}

Borgström B., I967. Partition of lipids between emulsified oil and micellar phases of glyceride-bile salt dispersions. J. Lipid Res., 8, 598-608.

Constantin M. J., Savary P., I965. Sur l'incorporation des chaînes stéariques dans les chylomicrons lymphatiques du rat, Bioch. Biophys. Acta, 106, 248-260.

Demarne Y., Sacguet E., Flanzy J., Garnier H., François A. C., 197o. Utilisation digestive apparente des acides gras chez le Rat axénique et le Rat holoxénique. Ann. Biol. anim. Bioch. Biophys., 10, $369-384$.

Demarne Y., Sacquet E., Flanzy J., Garnier H., François A. C., 1972. Influences cumulées de la caecectomie et de l'état axénique sur l'utilisation digestive apparente de la ration et des acides gras chez le Rat. Ann. Biol. anim. Bioch. Biophys., 12, I39-148.

Einarsson K., ig66. On the formation of hyodeoxycholic acid. J. Biol. Chem., 241, 534-539.

Grundy S. M., Ahrens E. H., Mietrinen T. A., i965. Quantitative isolation and gasliquid chromatographic analysis of total fecal bile acids. J. Lipid Res., 6, 397-410.

Gueguen L., Rombauts P., I96r. Dosage du sodium, du potassium, du calcium et du magnésium par spectrophotométrie de flamme dans les aliments, le lait et les excreta. Ann. Biol. anim. Bioch. Biophys., 1, 80-97.

Hofmann A. F., I96I. Micellar solubilization of fatty acids and monoglycerides in bile salt solutions. In : The enzymes of lipid metabolism. Proceedings of the sixth International Conference on the Biochemistry of Lipids, Marseille, 1960, P. Desnuelle, editor, I59-171.

Hofmans A. F., 1963. Further studies on the intraluminal phase of fat digestion : factors influencing the behaviour of fatty acids in bile-salt solutions. VI Congrès de Nutrition, Edimburg, 50r.

Hofmann A. F., I964. Thin layer chromatography of bile acids and their derivatives. In: JAMEs A., Morris L., Nere biochemical separations. D. Van Nostrand Co Ltd, London, 261-282.

Kellog T. F., I97I. Microbiological aspects of enterohepatic neutral sterol and bile acid metabolism. Fed. Proc., 30, 1808-1814.

Sacquet E., I971. Flore microbienne du tractus digestif et nutrition chez Rattus norvegicus. Thèse de Doctorat ès-Sciences. Université de Paris.

Sacquet E., Garnier H., Ionesco D., ig7o. Composition de la bile et du contenu de l'intestin grêle du Rat "axénique " en acides biliaires. C. R. Acad. Sc., 271, I809-181 2.

Savary P., Constantin M. J., I 666 . Sur la résorption des chaines éruciques et leur incorporation dans les chylomicrons lymphatiques du Rat. Biochim. Biophys. Acta, 125, I18-128.

Schiff E. R., Small N. C., Dietschy S. M., 1972. Characterization of the kinetics of the passive and active transport mechanisms for bile acid absorption in the small intestine and colon of the rat. J.clin. Invest., 51, I35 I-I 362 .

Shefer S., Hauser S., Bekersky I., Mosbach E. H., r969. Feedback regulation of bile acid biosynthesis in the rat. J. Lipid. Res., 10, 646-655.

Shimada K., Sutter V. I., Finegold S. M., I97o. Effect of bile and desoxycholate on gram-negative anaerobic bacteria. Appl. Microbiol, 20, 737-741.

Subbiah M. T., Kuksis A., I968. Alkaline solvent systems for thin-layer chromatography of bile acids. J. Lipid. Res., 9, 288-29o.

Stempfel R. S., Sidbury J. B., I964. Studies with the hydroxysteroid dehydrogenases. I. A simplified method for the enzymatic estimation of 3 and I7 hydroxysteroids. $J$. Clin. Endocr., 24, 367-372.

Van heijenoort Y., Sacouet E., Raibaud P., Demarne Y., Mathis C., 1972. Action de la flore microbienne sur le contenu de l'intestin grêle du rat en sels biliaires. $C$. $R$. Acad. Sc., 275, $271-274$. 